\title{
Erratum to: Spondyloenchondrodysplasia Due to Mutations in ACP5: A Comprehensive Survey
}

\author{
Tracy A. Briggs ${ }^{1,2} \cdot$ Gillian I. Rice $^{1} \cdot$ Navid Adib $^{3} \cdot$ Lesley Ades $^{4,5} \cdot$ Stephane Barete $^{6}$. \\ Kannan Baskar $^{7}$ - Veronique Baudouin ${ }^{8}$ - Ayse N. Cebeci ${ }^{9}$ - Philippe Clapuyt ${ }^{10}$. \\ David Coman ${ }^{11,12}$ - Lien De Somer ${ }^{13}$ - Yael Finezilber ${ }^{14}$ - Moshe Frydman ${ }^{14,15}$. \\ Ayla Guven ${ }^{9,16}$ - Sébastien Heritier ${ }^{17}$ - Daniela Karall ${ }^{18}$ - Muralidhar L. Kulkarni ${ }^{19}$. \\ Pierre Lebon $^{20}$ • David Levitt ${ }^{21}$ - Martine Le Merrer ${ }^{22}$ • Agnes Linglart ${ }^{23,24}$. \\ John H. Livingston ${ }^{25}$ • Vincent Navarro ${ }^{26}$ - Ericka Okenfuss ${ }^{27}$ - Anne Puel ${ }^{28}$. \\ Nicole Revencu ${ }^{29}$ - Sabine Scholl-Bürgi ${ }^{18}$ - Marina Vivarelli ${ }^{30}$ - Carine Wouters $^{31}$. \\ Brigitte Bader-Meunier $^{32,33}$ • Yanick J. Crow ${ }^{1,34}$
}

Published online: 29 April 2016

(C) Springer Science+Business Media New York 2016

\section{Erratum to: J Clin Immunol. 2016 Apr;36(3):220-34 DOI 10.1007/s10875-016-0252-y}

The original version of this article did not contain acknowledgements. These are presented below.

Acknowledgments We sincerely thank the participating families for the use of genetic samples and clinical information. We also thank Dr

The online version of the original article can be found at http://dx.doi.org/ 10.1007/s10875-016-0252-y.

Tracy A. Briggs

tracy.briggs@manchester.ac.uk

1 Manchester Centre for Genomic Medicine, Institute of Human Development, Faculty of Medical and Human Sciences, University of Manchester, Manchester, UK

2 St Mary's Hospital, Central Manchester University Hospitals NHS Foundation Trust, Manchester Academic Health Science Centre, Manchester, UK

3 Department of Rheumatology, The Lady Cilento Children's Hospital, Brisbane, Australia

4 Department of Clinical Genetics, The Children's Hospital at Westmead, Sydney, Australia

5 Discipline of Paedatrics and Child Health, The University of Sydney, Sydney, Australia

6 Dermatology Department, Pitie-Salpetriere Hospital, Paris, France

7 Creighton University, 2500 California Plaza, Omaha, NE 68178, USA
Banan Musallam-Jabbour, Israel and Dr Giuseppina Ognisanti, Sicily for providing clinical data. TAB acknowledges funding support from the National Institute for Health Research, the Academy of Medical Sciences, the Wellcome Trust, Medical Research Council, British Heart Foundation, Arthritis Research UK, Prostate Cancer UK and the Royal College of Physicians and the National Institute for Health Research Manchester Musculoskeletal Biomedical Research Unit. The views expressed in this publication are those of the author(s) and not necessarily those of the NHS, the National Institute for Health

8 Pediatric Nephrology Department, Robert Debré University Hospital- APHP, 48 boulevard Sérurier, 75019 Paris, France

9 Goztepe Educational and Research Hospital Pediatric Endocrinology Clinic, Istanbul, Türkiye

10 Pediatric Imaging Unit, Cliniques universitaires Saint-Luc, Université catholique de Louvain, Brussels, Belgium

11 Neuroscience Department, The Lady Cilento Children's Hospital, Brisbane, Australia

12 School of Medicine, Griffith University, Gold Coast, Australia

13 Pediatric Rheumatology, Department of Pediatrics, University Hospitals Leuven, B-3000 Leuven, Belgium

14 Danek Gertner Institute of Human Genetics, Chaim Sheba Medical Center, Tel Aviv, Israel

15 Sackler School of Medicine, Tel Aviv University, Tel Aviv, Israel

16 Amasya University Medical Faculty, Department of Pediatric Endocrinology, Istanbul, Türkiye 
Research or the Department of Health. Y.J.C. acknowledges the European Research Council (GA 309449: Fellowship), and a state subsidy managed by the National Research Agency (France) under the "Investments for the
Future" program bearing the reference ANR-10-IAHU-01. The authors would like to thank the National Heart, Lung, and Blood Institute (NHLBI) Exome Sequencing Project (ESP) database.
17 Department of Pediatric Hematology and Oncology, Trousseau Hospital, Assistance Publique-Hôpitaux de Paris (APHP), Paris, France

18 Clinic for Pediatrics I, Inherited Metabolic Disorders, Medical University of Innsbruck, Anichstr. 35, A-6020 Innsbruck, Austria

19 J. J. M. Medical College, Davangere, Karnataka 577004, India

20 Service de Virologie, AP-HP Hôpital Cochin, Paris, France

21 Department of Paediatrics, The Lady Cilento Children's Hospital, Brisbane, Australia

22 Centre de Référence des Maladies Osseuses Constitutionnelles et Institut Imagine, Hopital Necker 149 rue de Sevres, 75015 Paris, France

23 APHP, Bicêtre Paris Sud, Department of Pediatric Endocrinology and Diabetology for Children, 94270 Le Kremlin Bicêtre, France

24 Reference Center for Rare Disorders of the Mineral Metabolism and Plateforme d'expertise Paris Sud Maladies Rares, APHP, 94270 Le Kremlin Bicêtre, France

25 Department of Paediatric Neurology, Leeds Teaching Hospitals NHS Trust, Leeds, UK

26 Epilepsy Unit, Pitie-Salpetriere Hospital, Paris, France

27 Kaiser Permanente - Genetics, 1650 Response Rd, Sacramento, CA 95815, USA

28 Génétique Humaine des Maladies Infectieuses, INSERM UMR 1163, Université Paris Descartes Sorbonne Paris Cité, Institut Imagine, Pièce 421-B1, 24 boulevard du Montparnasse, 75015 Paris, France

29 Centre for Human Genetics, Cliniques universitaires Saint-Luc, Université catholique de Louvain, Brussels, Belgium

30 Division of Nephrology, IRCCS Bambino Gesu' Pediatric Hospital, Rome, Italy

31 Department of Microbiology and Immunology, Pediatric Immunology, KU Leuven, University of Leuven, Leuven, Belgium

32 Pediatric Immunology and Rheumatology Unit, Hôpital Necker, APHP, Paris, France

33 Institut Imagine, Paris, France

34 Laboratory of Neurogenetics and Neuroinflammation, Institut Imagine, 24 boulevard du Montparnasse, 75015 Paris, France 